\title{
Accessory left biliary duct draining into the lesser curve of the stomach
}

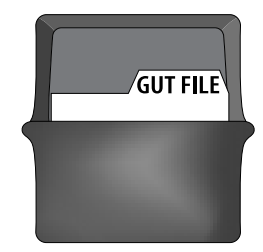

\section{CASE REPORT}

A 32 year old woman presented to a hospital in North America in November 1996 with upper abdominal pain and pyrexia. Liver function tests and ultrasound scan of the abdomen were normal. Gastroscopy demonstrated an abnormal opening on the lesser curve of the stomach which on barium meal investigation appeared as a sinus tract extending superiorly (fig lA). A computed tomography (CT) scan performed showed minimal aerobilia in the left liver but no other abnormality. At laparoscopy, a well formed tract was identified running from the gastric lesser curve towards the left side of the hilum of the liver. This was excised using a linear stapler. Histology revealed biliary type epithelium in the excised segment of the tract.

She presented to this unit in August 1997 with further episodes of abdominal pain and fever. A CT scan was performed and this showed mild dilatation of the left intrahepatic ducts. Endoscopic retrograde cholangiopancreatography (ERCP) demonstrated stenosis of the left hepatic duct with a large proximal concretion (fig 1B). In addition, there was a diverticulum passing inferiorly from above the stenosis which corresponded to the upper end of the previously divided tract. Laparotomy and left
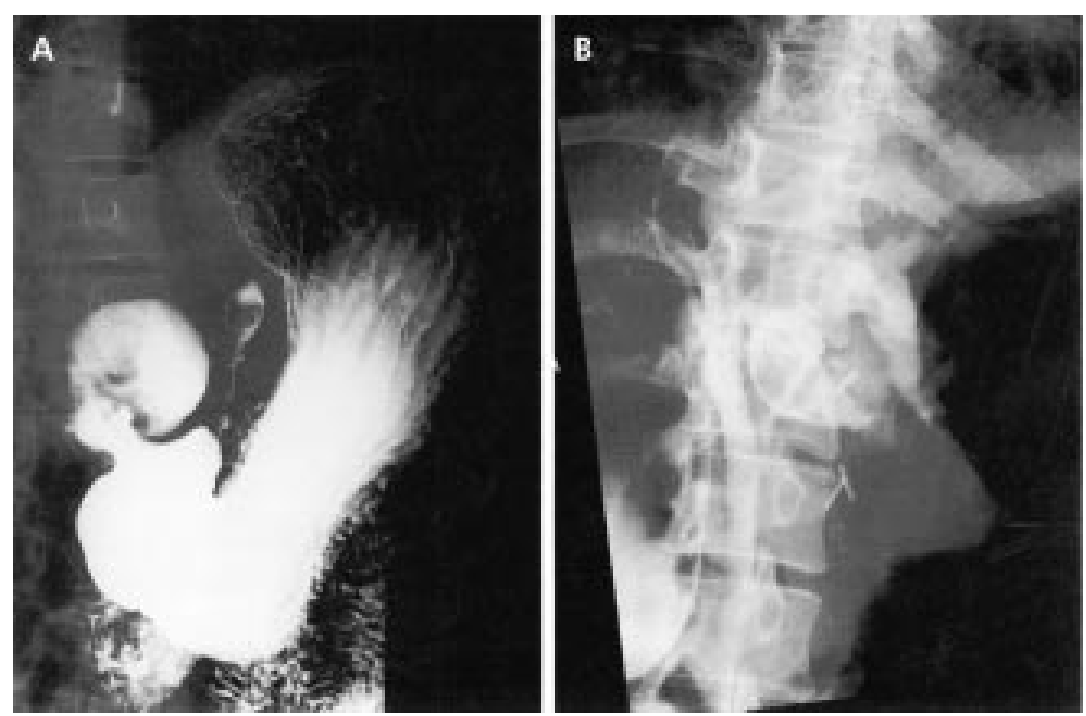

Figure 1 (A) Barium meal showing sinus tract extending from the lesser curve of the stomach. (B) Endoscopic retrograde cholangiopancreatography (ERCP) demonstrating stenosis of the left hepatic duct with proximal dilatation. hepatectomy were performed. The resected specimen revealed stenosis of the left hepatic duct $1 \mathrm{~cm}$ above the hepatic duct confluence. The left intrahepatic ducts were grossly distended and filled with sludge distal to the stenosis.

This patient made an uneventful recovery and remains well four years since surgery.

\section{DISCUSSION}

The incidence of aberrant anatomy of bile ducts varies from 16 to $31 \% .^{12}$ Aberrant or accessory hepatic ducts from the right side of the liver are more common with drainage into the right hepatic duct, cystic duct, and occasionally into the gall bladder. There have been isolated reports of separate entry of the right and left hepatic ducts into the duodenum. There are no reported cases in the literature showing drainage of a bile duct into the lesser curve of the stomach. ${ }^{34}$

The presence of an aberrant duct in the free edge of left triangular ligament has also been reported. ${ }^{5}$ Distal communication of this structure with the gastrointestinal tract is not recorded and this anomaly probably represents distal extension of atrophic hepatic elements into the left triangular ligament rather than a true functioning duct.

In the present case, the structure divided laparoscopically was most likely

\section{REFERENCES}

1 Adams DB. The importance of extra-hepatic biliary anatomy in preventing complications at laparoscopic cholecystectomy. Surg Clin North Am 1993;73:861-71

2 Crist DW, Gadacz TR. Laparoscopic anatomy of the biliary tree. Surg Clin North Am 1993;73:785-98

3 Gore RM, Ghahremanio GG, Fernbach SK Gallbladder and biliary tract: Anomalies and anatomical variants. Textbook of gastrointestinal radiology. Philadelphia: WB Saunders, 1994

4 Pellegrini CA, Quan-Yang D. Gallbladder and Biliary tree: Anatomy and structural anomalies. Textbook of gastroenterology. Philadelphia: JB Lippincott Co, 1995.

5 Iso Y, Kusaba I, Matsumata T, et al. Postoperative bile peritonitis caused by division of an aberrant bile duct in the left triangular ligament of the liver. Am J Gastroenterol 1996;91:2428-30 